\title{
Virulence of ten serogroups of Clostridium difficile in hamsters
}

\author{
M. DELMÉE and V. AVESANI
}

\section{University of Louvain, Microbiology Unit, Avenue Hippocrate, 54 B-5490, B-1200 Brussels, Belgium}

\begin{abstract}
Summary. A slide agglutination technique identifying 10 serogroups of Clostridium difficile (A,B,C,D,F, G,H,I,K and X) has been described previously. In this study, we have used the hamster to compare the ability of the 10 serogroup reference strains to colonise and produce disease. Groups of four hamsters were each given a single intraperitoneal injection of either clindamycin or cefoxitin, and an oral challenge dose of $C$. difficile. The time taken to establish faecal colonisation and the length of survival after colonisation were monitored. All hamsters treated with cefoxitin became colonised by day 3 and those challenged with the cytotoxigenic strains of serogroups A,C, $\mathrm{H}$ and $\mathrm{K}$ developed colitis and died. Among those challenged with the non-toxigenic strains of groups B,D,I and $X$ and the toxigenic strains of groups $F$ and $\mathrm{G}$, faecal colonisation was established without signs of disease. This demonstrates that there are differences in virulence even among toxigenic strains of $C$. difficile. The same phenomenon was observed after treatment with clindamycin but the pattern of colonisation was quite different with some strains. In the hamsters challenged with toxigenic strains of groups $\mathrm{C}$ and $\mathrm{K}$ and non-toxigenic strains of groups $\mathrm{D}$ and $\mathrm{I}$, which are highly resistant to clindamycin, the response was the same as with cefoxitin. The results were different with strains which were susceptible to clindamycin. Some animals became colonised much later than those treated with cefoxitin but the mortality was similar. The remaining animals became colonised by day 3 , not by the challenge organism but by another strain which always belonged to serogroup D or $\mathrm{C}$; the source of these organisms was thought to be either previously undetected faecal carriage or the environment. Four animals infected by a toxigenic serogroup$\mathrm{C}$ strain died. Another five, colonised by a non-toxigenic serogroup-D strain survived even though they had been challenged with a strain of a virulent serogroup. This protective effect and the differences in the potential to colonise after clindamycin therapy may warrant further investigation in man.
\end{abstract}

\section{Introduction}

A serogrouping technique by which 10 serogroups (A,B,C,D,F,G,H,I,K and X) of Clostridium difficile are identified has been described previously. ${ }^{1,2}$ So far, more than 3000 strains from various sources have been typed by this method and $96 \%$ have been allocated to one of the 10 serogroups (unpublished data). Several epidemiological studies have shown that not all serogroups are equally pathogenic. ${ }^{3-5}$ Isolates of serogroups A,C,H and $\mathrm{K}$ that produced cytotoxin were most commonly found in cases of antibiotic associated diarrhoea. Among these, serogroup $C$ appeared most prevalent and was implicated in most of the outbreaks that we reported. ${ }^{3,4}$ The non-cytotoxigenic isolates of serogroup D were isolated from asymptomatic adults

Received 8 Aug. 1989; revised version accepted 5 March 1990. or children. Strains belonging to serogroups B,F,G,I and $\mathrm{X}$ were isolated from asymptomatic children or neonates. One of the most interesting observations was that most of the strains belonging to serogroups $F$ and $G$ were toxigenic and cytotoxin were usually found in the faeces of asymptomatic carriers.

In 1968, Small reported that hamsters given a subcutaneous injection of lincomycin often died with enterocolitis. ${ }^{6}$ Later it was found that $C$. difficile and cytotoxin were present in the faeces of hamsters with the disease. ${ }^{7-9}$ Since those first reports, the hamster model has yielded important information on aspects of pathogenicity, epidemiology and treatment. ${ }^{10-12}$ In this study we have compared the pathogenicity for hamsters of strains belonging to the 10 different serogroups of $C$. difficile. 


\section{Materials and methods}

\section{Strains of C. difficile}

Eleven strains were used: reference strains of the 10 serogroups of $C$. difficile $e^{1,2}$ and strain 11181 , belonging to serogroup $\mathbf{K}$, which had been isolated from a case of pseudomembranous colitis (PMC). Reference numbers, production of cytotoxin and source of each strain are indicated in table $\mathrm{I}$.

\section{Preparation of inoculum for animal experiments}

Bacteria were grown for $18 \mathrm{~h}$ in $10 \mathrm{ml}$ of a pre-reduced liquid medium (LM) at $37^{\circ} \mathrm{C}$ in anaerobic conditions, ${ }^{1}$ harvested by centrifugation at $2000 \mathrm{~g}$ for $5 \mathrm{~min}$ and resuspended in $1 \mathrm{ml}$ of saline. This suspension was mixed with $1 \mathrm{ml}$ of a 7-day culture in semi-solid thioglycollate medium, to give a suspension containing $10^{7}-10^{8} \mathrm{cfu} / \mathrm{ml}$.

\section{Isolation of C. difficile from hamsters and detection of cytotoxin}

Faecal pellets collected in a sterile container were emulsified in $4 \mathrm{ml}$ of sterile saline; $0.1 \mathrm{ml}$ was inoculated on to cycloserine-cefoxitin-fructose agar ${ }^{13}$ incorporating sodium taurocholate $0.01 \% \mathrm{w} / \mathrm{v}$. Cultures were incubated anaerobically for $48 \mathrm{~h}$ at $37^{\circ} \mathrm{C}$. C. difficile isolates were identified by colonial morphology and gas liquid chromatography. Faecal filtrate was examined for $C$. difficile cytotoxin (toxin B) by inoculation of confluent monolayers of HeLa cells with specific neutralisation of $C$. sordellii antitoxin (Wellcome sa, Alost, Belgium). Production of toxin A (enterotoxin) was not investigated. In this report, the terms toxin and toxigenic always refer to cytotoxin.

\section{Serogrouping}

Serogrouping was by slide agglutination ${ }^{1}$ with 10 rabbit antisera designated A,B,C,D,F,G,H,I,K and X. Two colonies were serogrouped from each positive culture of faeces. Cytotoxin production in vitro was tested with filtrates of 48-h liquid cultures.

\section{Polyacrylamide gel electrophoresis (PAGE)}

Isolates belonging to serogroup A were further distinguished by PAGE. Briefly, cells of an 18-h culture in LM were sonicated, treated with sodium dodecyl sulphate $10 \%$ and 2-mercapto-ethanol $15 \%$, heated at $100^{\circ} \mathrm{C}$ for $5 \mathrm{~min}$, and proteins were separated by electrophoresis on polyacrylamide gel. After staining with Coomassie Blue, the profiles were compared with those of reference strains. Twelve different PAGE profiles designated A1$A 12$ are currently recognised within serogroup $A$ whereas strains from each of the other serogroups have a single group-specific profile. The W1194 reference strain of serogroup A used in our experiments had profile A1. ${ }^{2}$

\section{Antibiotics}

Clindamycin phosphate was supplied by Upjohn Co. (Puurs, Belgium) and cefoxitin by Merck Sharp and Dohme (Brussels, Belgium). For administration to hamsters, solutions contained clindamycin $15 \mathrm{mg} / \mathrm{L}$ or cefoxitin $30 \mathrm{mg} / \mathrm{L}$.

\section{Minimum inhibitory concentrations (MIC) of antimicrobial agents}

These were determined by an agar dilution technique ${ }^{14}$ with multipoint inoculation of $10^{5} \mathrm{cfu}$ on WilkinsChalgren agar supplemented with haemin and menadione and containing antimicrobial concentrations of $0.5-$ $256 \mathrm{mg} / \mathrm{L}$.

\section{Animal experiments}

Adult Syrian hamsters were housed individually in isolator cages with air filters fitted in their lids. Cages, food, water bottles and bedding were autoclaved in sealed bags before use.

Faecal pellets from each hamster were cultured for $C$. difficile before each experiment. Animals yielding positive cultures were discarded. Groups of four animals treated with one of the antibiotics were challenged with each test strain. On day 0 , hamsters were given a single intraperitoneal (i.p.) injection of $0.5 \mathrm{ml}$ of clindamycin $(7.5 \mu \mathrm{g})$ or cefoxitin $(15 \mu \mathrm{g})$. Animals were then given $1 \mathrm{ml}$ of the $C$. difficile suspension by gastric inoculation and $1 \mathrm{ml}$ of the same suspension was added to $20 \mathrm{ml}$ of the drinking water. After $72 \mathrm{~h}$, the drinking water was replaced by fresh water in sterilised bottles. On and after day 3, faecal pellets from each animal were collected daily for 21 days and the clinical condition was noted. Two groups of four hamsters were usually investigated at the same time and, for each series, one additional hamster given an antimicrobial injection only was used as a control.

Of 49 hamsters treated with cefoxitin and 48 treated with clindamycin, five control hamsters received cefoxitin alone and four received clindamycin alone. Groups of four hamsters treated with each antimicrobial agent each received one of the 11 different strains of $C$. difficile.

\section{Results}

\section{Antimicrobial susceptibility tests}

Results are given in table $\mathbf{I}$.

\section{Cefoxitin experiments}

The number of deaths in each group of hamsters is shown in table II.

In the first faecal cultures, performed on day 3 , C. difficile was isolated from all but five animalsfour of the five control animals and one animal challenged with the group D strain. Faeces from 
Table I. Sources and characteristics of $C$. difficile strains

\begin{tabular}{rrrrrrr}
\hline \multirow{2}{*}{$\begin{array}{c}\text { Strain } \\
\text { no. }\end{array}$} & ATCC no. & Serogroup & $\begin{array}{c}\text { Cytotoxin } \\
\text { production } \\
\text { in vitro }\end{array}$ & Source & \multicolumn{2}{c}{ MIC (mg/L) of } \\
\cline { 5 - 7 } clindamycin & cefoxitin \\
\hline W1194 & 43594 & A & + & PMC & 1 & 64 \\
1351 & 43593 & B & - & Neonate* & 2 & 64 \\
545 & 43596 & C & + & PMC & $>512$ & 128 \\
3232 & 43597 & D & - & Adult* & $>512$ & 64 \\
1470 & 43598 & F & + & Neonate* & 2 & 64 \\
2022 & 43599 & G & + & Neonate* & 1 & 64 \\
2149 & 43600 & H & + & PMC & 1 & 64 \\
7322 & 43601 & I & - & Neonate* & $>512$ & 64 \\
4811 & 43602 & K & - & Neonate* & $>512$ & 64 \\
5036 & 43603 & X & - & Neonate* & $0 \cdot 5$ & 64 \\
11181 & $\ldots$ & K & + & PMC & $>512$ & 64 \\
\hline
\end{tabular}

PMC, pseudomembranous colitis.

*Asymptomatic.

the latter yielded $C$. difficile on day 4 . The faecal cultures of one control remained negative until the end of the experiment on day 21 , but four controls became colonised on days 3, 4, 4 and 6 .

Cytotoxin was detected in all culture-positive faeces when the challenge had been a known toxigenic strain. The strains isolated from faeces were the same as those which had been administered orally. Two control animals were colonised by day 4 and day 6 with a toxigenic serogroup-A strain; both died on day 10 and both isolates had PAGE profile A6. The third was colonised by day

Table II. Summary of results with hamsters treated with cefoxitin or clindamycin and challenged with $C$. difficile strains

\begin{tabular}{rrll}
\hline & & \multicolumn{2}{c}{$\begin{array}{c}\text { Number of deaths (day of } \\
\text { death) in groups* of hamsters } \\
\text { treated with }\end{array}$} \\
$\begin{array}{rrll}\text { Strain } \\
\text { no. }\end{array}$ & $\begin{array}{c}\text { Serogroup (cyto- } \\
\text { toxin production }\end{array}$ & \multicolumn{2}{c}{ in vitro) } \\
\cline { 4 - 4 } W1194 & cefoxitin & clindamycin \\
1351 & $\mathrm{~A}(+)$ & $4(5,5,6,7)$ & $3(10,12,12)$ \\
545 & $\mathrm{~B}(-)$ & 0 & $2(5,5)$ \\
3232 & $\mathrm{C}(+)$ & $4(4,4,5,5)$ & $4(4,4,5)$ \\
1470 & $\mathrm{D}(-)$ & 0 & 0 \\
2022 & $\mathrm{~F}(+)$ & $1(20)$ & $2(13,17)$ \\
2149 & $\mathrm{G}(+)$ & 0 & $1(5)$ \\
7322 & $\mathrm{H}(+)$ & $4(4,4,5,7)$ & $3(4,10,13)$ \\
4811 & $\mathrm{I}(-)$ & 0 & 0 \\
11181 & $\mathrm{~K}(-)$ & 0 & 0 \\
5036 & $\mathrm{~K}(+)$ & $4(7,7,10,14)$ & $4(7,7,10,10)$ \\
controls & $\mathrm{X}(-)$ & 0 & 0 \\
& $\ldots$ & $3(6,10,10)$ & $2(7,13)$ \\
& & &
\end{tabular}

*Four hamsters in each group except for five in the cefoxitin control group.
4 with a non-toxigenic group-D strain and remained an asymptomatic carrier of this strain until the end of the experiment and the fourth animal was colonised by day 3 with a toxigenic group-C strain and died on day 6.

The median day of death of animals challenged with a toxigenic strain of group $\mathrm{A}, \mathrm{C}$ or $\mathrm{H}$ ranged from 4.5 to 5.75 days. Animals given the toxigenic strain of group $\mathrm{K}$ generally died later (9.5 days), but one had a positive faecal culture on day 3 , negative cultures on days 4-6 and positive cultures from day 7 until death on day 14 .

Diarrhoea usually correlated with presence of cytotoxin in faeces except with strains of groups $G$ or $F$. One animal challenged with the group-G strain had diarrhoea on days 6-11 but then recovered and remained well until day 21 . The animal that died on day 20 and had been challenged with the serogroup-F strain did not have diarrhoea.

All animals which survived continued to have positive faecal cultures until day 21 , the last day of the experiment. Cytotoxin was detected throughout the experiment in those challenged with toxigenic strains of group $\mathrm{G}$ or $\mathrm{F}$.

\section{Clindamycin experiments}

The number of deaths in each group is given in table II.

Results obtained after challenge with clindamycin-resistant strains belonging to groups C,D,I and $\mathrm{K}$ (toxigenic and non-toxigenic) were similar to those in hamsters treated with cefoxitin. All animals were colonised by day 3 with the strain which had been administered. Animals challenged with the group-C strain died after $4,4,4$ and 5 days and those 
given the toxigenic group-K strain (strain 11181) died after 7,7,10 and 10 days. All of the 12 animals which received strains of groups D,I and $K$ (nontoxigenic) remained well.

The behaviour of clindamycin-susceptible strains from groups $A, B, F, G, H$ and $X$ in clindamycintreated hamsters was different from that in cefoxitin-treated animals. Fourteen hamsters had delayed faecal colonisation when compared with the cefoxitin experiments but in each case serogrouping confirmed that the strain was of the serogroup that had been administered. The first positive faecal culture was recorded between day 7 and day 12 . Death or survival depended on the serogroup of the strain. Ten hamsters were colonised by a strain of $C$. difficile of a serotype different from that of the strain which had been administered (table III). Four animals were already colonised on day 3 by toxigenic clindamycin-resistant serogroup-C strains; one animal died on day 4, and the rest on day 5. Five hamsters became colonised by nontoxigenic clindamycin-resistant group-D strains on day 3,4 or 5 . All five remained carriers of group-D strains and survived until day 21 . A hamster which had been challenged with a group-F strain was colonised with the group-F strain on day 4 although cytotoxin was not detected in faeces. On and after day 5 , cytotoxin was detected in faeces and a strain belonging to group $\mathrm{A}$ was isolated. The animal died on day 13 . The isolate had a PAGE profile $A 6^{2}$ and was susceptible to clindamycin.

Three control animals became colonised with $C$. difficile. One with a toxigenic group-C strain on day 4 died on day 7 . The second with a toxigenic group-
A strain (PAGE profile A6) was colonised by day 10 and died on day 13 . The third was colonised with a group-D strain by day 4 and remained a carrier until the end of the experiment.

\section{Discussion}

This work clearly demonstrated differences of pathogenicity among strains belonging to the 10 different serogroups of $C$. difficile; these differences correlated with observations of serogroup-related differences in pathogenicity in man. Toxigenic strains belonging to groups $\mathrm{A}, \mathrm{C}, \mathrm{H}$ and $\mathrm{K}$ killed all animals a few days after colonisation. These groups have also been implicated in cases of colitis in man. Toxigenic isolates belonging to groups $F$ and $G$ are usually isolated from asymptomatic neonates or children ${ }^{1,5}$ and strains belonging to these groups were non-pathogenic to hamsters even though cytotoxin was produced in vivo. Finally, nontoxigenic strains belonging to groups $\mathrm{B}, \mathrm{D}, \mathrm{I}, \mathrm{K}$ and $X$ were non-pathogenic for hamsters. These serogroups seem to be non-pathogenic for man. Pathogenicity was linked to cytotoxin production except with groups $F$ and $G$.

Colonisation by non-pathogenic serotypes may explain why infants remain asymptomatic despite the presence of faecal cytotoxin. ${ }^{15-17}$ Other theories have proposed a lack of toxin receptors on the intestinal mucosa or inhibitors of toxin in the faeces. Reports of cases of PMC in young children $^{18,19}$ and a recent observation of PMC in a 6-month-old child due to a toxigenic serogroup-C strain (Delmée, unpublished observations) help to

Table III. Selected results of faecal cultures and cytotoxin assays for clindamycin-treated hamsters which were colonised with a strain of $C$. difficile different from their challenge strain

\begin{tabular}{|c|c|c|c|c|c|c|c|c|c|}
\hline \multirow{2}{*}{$\begin{array}{l}\text { Serogroup (cytotoxin } \\
\text { production in vitro) of } \\
\text { the challenge strain }\end{array}$} & \multicolumn{9}{|c|}{ Results of culture* (and cytotoxin assay) of faecal pellets collected on day } \\
\hline & 3 & 4 & 5 & 7 & 10 & 12 & 13 & 17 & 21 \\
\hline $\mathrm{A}(+)$ & $-(-)$ & $-(-)$ & $\mathrm{D}(-)$ & $\mathrm{D}(-)$ & $\mathrm{D}(-)$ & $\mathbf{D}(-)$ & $\mathrm{D}(-)$ & $\mathbf{D}(-)$ & $\mathbf{D}(-)$ \\
\hline $\mathrm{B}(-)$ & $\mathrm{D}(-)$ & $\mathrm{D}(-)$ & $\mathrm{D}(-)$ & $\mathrm{D}(-)$ & $\mathrm{D}(-)$ & $\mathrm{D}(-)$ & $\mathrm{D}(-)$ & $\mathrm{D}(-)$ & $\mathbf{D}(-)$ \\
\hline B (-) & $\mathrm{C}(+)$ & $\mathrm{C}(+)$ & $\mathrm{C}(+) \dagger$ & & & & & & \\
\hline $\mathrm{B}(-)$ & $C(+)$ & $C(+)$ & $\mathrm{C}(+) t$ & & & & & & \\
\hline $\mathbf{F}(+)$ & $-(-)$ & $-(-)$ & $F(-)$ & $\mathbf{A}(+)$ & $\mathrm{A}(+)$ & $\mathrm{A}(+)$ & $\mathrm{A}(+) \dagger$ & & \\
\hline$G(+)$ & $\mathrm{C}(+)$ & $C(+)$ & $\mathrm{C}(+) \dagger$ & & & & & & \\
\hline $\mathbf{H}(+)$ & $-(-)$ & $\mathrm{D}(-)$ & $D(-)$ & $\mathrm{D}(-)$ & $\mathrm{D}(-)$ & $\mathrm{D}(-)$ & $\mathrm{D}(-)$ & $\mathrm{D}(-)$ & $\mathrm{D}(-)$ \\
\hline $\mathbf{H}(+)$ & $\mathrm{C}(+)$ & $\mathrm{C}(+) \dagger$ & & & & & & & \\
\hline$X(-)$ & $-(-)$ & $\mathrm{D}(-)$ & $\mathrm{D}(-)$ & $\mathbf{D}(-)$ & $\mathrm{D}(-)$ & $\mathrm{D}(-)$ & $\mathrm{D}(-)$ & $\mathrm{D}(-)$ & $\mathrm{D}(-)$ \\
\hline$X(-)$ & $-(-)$ & $-(-)$ & $\mathrm{D}(-)$ & $\mathbf{D}(-)$ & $\mathrm{D}(-)$ & $\mathbf{D}(-)$ & $D(-)$ & $D(-)$ & $\mathbf{D}(-)$ \\
\hline
\end{tabular}

${ }^{*}$ C. difficile not isolated; A, C, D, F, isolation of strain of indicated group.

†Day of death. 
substantiate the first hypothesis. Borriello et al. have reported that not all cytotoxigenic strains of C. difficile are equally virulent in the hamster model $;{ }^{20}$ two of the less virulent isolates studied by these authors were from asymptomatic infants and another was from the ward environment of a neonatal unit, but these isolates were not serotyped. We believe that serogrouping may be useful for discriminating pathogenic from non-pathogenic strains, especially when they are isolated from neonates or children. However, further studies are needed to confirm that all strains within a serogroup have similar virulence.

One possible explanation for these differences in pathogenicity could be a discrepancy between production of toxins A and B. Our assay detected cytotoxin (toxin B) only. However, toxin A, an enterotoxin, has been shown to be the main determinant of pathogenicity in hamsters. Lyerly et al. have proposed that toxins $\mathrm{A}$ and $\mathrm{B}$ may act synergically and tissue damage due to toxin A may predispose to the action of toxin $\mathrm{B}^{21}$ Absence of toxin-A production in strains from groups $F$ and $G$ could explain their lack of pathogenicity. However, Libby et al. have reported that toxin A may be present in asymptomatic infants. ${ }^{22}$ Borriello et al. found no correlation between virulence and production of toxins A and B in vitro, but a correlation between virulence and the ability to generate high levels of toxin $\mathrm{A}$ in vivo. ${ }^{20}$

Clindamycin and cefoxitin are antimicrobial drugs which are commonly associated with antibiotic associated colitis. Both have been used in the hamster model. ${ }^{8,23,24}$ Previous reports have shown that clindamycin-susceptible $(\mathrm{MIC}<4 \mathrm{mg} / \mathrm{L})$ strains of $C$. difficile behave differently from highly resistant (MIC $>512 \mathrm{mg} / \mathrm{L}$ ) strains. ${ }^{25,26} \mathrm{Re}$ cently, we have demonstrated that resistant groups of strains can be found only within serogroups $\mathrm{C}, \mathrm{D}, \mathrm{I}$ and $\mathrm{K}^{27}$ Conversely, all $C$. difficile are relatively resistant to cephalosporins (MICs 64$128 \mathrm{mg} / \mathrm{L})^{25}$

The results obtained with the clindamycinresistant strains of groups $\mathrm{C}, \mathrm{D}, \mathrm{I}$ and $\mathrm{K}$ were identical to those obtained with the cefoxitintreated hamsters. A proportion of hamsters which had been treated with clindamycin and challenged

\section{REFERENCES}

1. Delmée $\mathbf{M}$, Homel $\mathbf{M}$, Wauters G. Serogrouping of Clostridium difficile strains by slide agglutination. J Clin Microbiol 1985; 21 : 323-327.

2. Delmée M, Laroche Y, Avesani V, Cornelis G. Comparison of serogrouping and polyacrylamide gel electrophoresis with one of the clindamycin-susceptible serogroups, was colonised more slowly in comparison with animals in the cefoxitin experiment. This suggests that colonisation resistance was due to the continued presence of an inhibitory concentration of antibiotic in the intestine for several days after injection of the drug. It is possible that spores of $C$. difficile, which are part of the inoculum, persisted in the gut and colonised the animal when antimicrobial concentrations decreased. Despite a delay in colonisation, the virulence of each serogroup was similar to that observed in cefoxitin experiments.

Some of the clindamycin-treated hamsters which were challenged with clindamycin-sensitive strains of $C$. difficile were colonised rapidly by a different strain of $C$. difficile belonging to group $\mathrm{C}$ or D (table III). Thus some hamsters challenged with clindamycin-susceptible strains behaved in the same way as some of the control animals. Seven of the nine controls also became colonised, with $C$. difficile from previously undetected faecal carriage or from the environment. Some of the isolates from the control animals belonged to group A but had a PAGE profile different from that of the group-A strain used in our challenge inoculum. Animals which were already colonised by serogroup-C strains died whilst those colonised by serogroup-D strains survived. Colonisation by clindamycinresistant non-toxigenic serogroups such as group D appeared to protect animals. This could have important clinical implications. Wilson and Sheagren ${ }^{28}$ first showed that colonisation with a non-toxigenic strain of $C$. difficile prior to challenge with toxigenic $C$. difficile was associated with $93 \%$ survival of hamsters compared with $21 \%$ survival of control animals. Borriello et al. proposed that the protection afforded by the non-toxigenic strains may be due to competition for ecological niches. ${ }^{29}$ Our data show that differences in susceptibility to clindamycin influences the time for colonisation to be established. It is possible that non-toxigenic clindamycin-resistant group-D strain administered to patients before clindamycin therapy might protect them against subsequent colonisation by pathogenic serogroups of $C$. difficile.

We thank Jeff Edwards for critical review of the manuscript.

for typing Clostridium difficile. J Clin Microbiol 1986; 24: 991-994.

3. Delmée M, Bulliard G, Simon G. Application of a technique for serogrouping Clostridium difficile in an outbreak of antibiotic-associated diarrhoea. J Infect 1986; 13: 5-9.

4. Delmée M, Vandercam B, Avesani V, Michaux JL. 
Epidemiology and prevention of Clostridium difficile infections in a leukemia unit. Eur J Clin Microbiol 1987; 6: 623-627.

5. Delmée M, Verellen G, Avesani V, Francois G. Clostridium difficile in neonates: serogrouping and epidemiology. Eur J Pediatr 1988; 147: 36-40.

6. Small JD. Fatal enterocolitis in hamster given lincomycin hydrochloride. Lab Anim Care 1968; 18: 411-420.

7. Bartlett JG, Chang TW, Moon N, Onderdonk AB. Antibiotic-induced lethal enterocolitis in hamsters: studies with eleven agents and evidence to support the pathogenic role of toxin-producing clostridia. Am J Vet Res 1978; 39: 1525-1530.

8. Larson HE, Price AB, Honour P, Borriello SP. Clostridium difficile and the aetiology of pseudmembranous colitis. Lancet 1978; 1 : 1063-1066.

9. Lusk RH, Fekety R, Silva J, Browne RA, Ringler DH, Abrams GD. Clindamycin-induced enterocolitis in hamsters. J Infect Dis 1978; 137 : 464-475.

10. Boon RJ, Beale AS. Studies with temocillin in hamster model of antibiotic-associated colitis. Antimicrob Agents Chemother 1985; 27 : 980-981.

11. Rolfe RD, Iaconis JP. Intestinal colonization of infant hamsters with Clostridium difficile. Infect Immun 1983; 42: $480-486$.

12. Weinberg DS, Fernandes PB, Kao CC, Clark JM, Bonner DP, Sykes RB. Evaluation of aztreonam, cefoperazone, latamoxef and ceftazidime in the hamster colitis model. $J$ Antimicrob Chemother 1986; 18: 729-745.

13. George WL, Sutter VL, Citron D, Finegold SM. Selective and differential medium for isolation of Clostridium difficile. J Clin Microbiol 1979; 9: 214-219.

14. Sutter VL, Barry AL, Wilkins TD, Zabransky RJ. Collaborative evaluation of a proposed reference dilution method of susceptibility testing of anaerobic bacteria. Antimicrob Agents Chemother 1979; 16: 495-502.

15. Donta ST, Meyers MG. Clostridium difficile toxin in asymptomatic neonates. J Pediatr 1982; 100: 431434.

16. Mardh P-A, Helin I, Colleen I, Oberg M, Holst E. Clostridium difficile toxin in faecal specimens of healthy children and children with diarrhoea. Acta Paediatr Scand 1982; 71 : 275-278.

17. Rietra PJGM, Slaterus KW, Zanen HC, Meuwissen SGM.
Clostridial toxin in faeces of healthy infants. Lancet $1978 ; 2: 319$.

18. Adler SP, Chandrika T, Berman WF. Clostridium difficile associated with pseudomembranous colitis. Occurrence in a 12-week-old infant without prior antibiotic therapy. Am J Dis Child 1981; 135: 820-822.

19. Mandal BK, Watson B, Ellis M. Pseudomembranous colitis in a 5-week-old infant. $\mathrm{Br}$ Med J 1982; 284: 345-346.

20. Borriello SP, Ketley JM, Mitchell TJ et al. Clostridium difficile-a spectrum of virulence and analysis of putative virulence determinants in the hamster model of antibiotic-associated colitis. J Med Microbiol 1987; 24: 53-64.

21. Lyerly DM, Saum KE, MacDonald DK, Wilkins TD. Effects of Clostridium difficile toxins given intragastrically to animals. Infect Immun 1985; 47: 349-352.

22. Libby JM, Donta ST, Wilkins TD. Clostridium difficile toxin $A$ in infants. $J$ Infect Dis $1983 ; 148: 606$.

23. Ebright JR, Fekety R, Silva J, Wilson KH. Evaluation of eight cephalosporins in hamster colitis model. Antimicrob Agents Chemother 1981; 19: 980-986.

24. Toshniwal R, Silva J, Fekety R, Kim KH. Studies on the epidemiology of colitis due to Clostridium difficile in hamsters. J Infect Dis 1981 ; 143: 51-54.

25. Ensminger PW, Counter FT, Thomas LJ, Lubbehusen PP. Susceptibility, resistance development, and synergy of antimicrobial combinations against Clostridium difficile. Curr Microbiol 1982; 7: 59-62.

26. Nakamura $S$, Nakashio $S$, Mikawa $M$, Yamakawa $K$, Okumura S, Nishida S. Antimicrobial susceptibility of Clostridium difficile from different sources. Microbiol Immunol 1982; 26: 25-30.

27. Delmée $\mathbf{M}$, Avesani V. Correlation between serogroup and susceptibility to chloramphenicol, clindamycin, erythromycin, rifampicin and tetracycline among 308 isolates of Clostridium difficile. J Antimicrob Chemother 1988; 22: 325-331.

28. Wilson KH, Sheagren JN. Antagonism of toxigenic Clostridium difficile by nontoxigenic $C$. difficile. $J$ Infect $D$ is 1983; 147: 733-736.

29. Borriello SP, Barclay FE. Protection of hamsters against Clostridium difficile ileocaecitis by prior colonisation with non-pathogenic strains. J Med Microbiol 1985; 19: 339-350. 